Ann. Biol. anim. Bioch. Biophys., I962, 2 (I), г3-16.

\title{
FÉCONDATION in vitro EN PRÉSENCE DE COLCHICINE ET POLYPLOIDIE EXPERIMENTALE CHEZ LE LAPIN
}

\author{
Ondine BOMSEL-HELMREICH et C. TIHIBAUL'T \\ avec la collaboration technique de Micheline GÉRARD \\ Station de Recherches de Physiologie animale, \\ (''nite national de Recherches zootechniques, Jouy-en-Josas (Seine-et-Oise).
}

En faisant agir la colchicine au moment de l'émission du deuxième globule polaire ou au moment de la première division de segmentation on peut espérer obtenir des œufs de Mammifères tri- ou tétraploïdes.

C'est dans ce but que HÄGGvis' et BANE ( $1950, a$ et $b$ ) mélangèrent de la colchicine au sperme inséminé et crurent obtenir des lapereaux polyploïdes d'après la taille des érythrocytes et des spermatozoïdes. L'analyse chromosomique de ces animaux par MELANDER (I950) posa des problèmes sans autoriser une conclusion. Mais MELANDER (I95I) répéta l'expérience chez la Truie en décrivant des mitoses triploïdes. En utilisant de même la voie utérine, pour introduire la colchicine, PORPACZY, GYORFFY, FARAGO et VASKuTTI (I959) ne trouvèrent aucun des agneaux, provenant de 3 I Brebis, polyploïdes.

Un autre groupe d'auteurs chercha à introduire la colchicine au niveau de l'œuf par injections intrapéritonéales. WALDO et WrMSATT (I945) n'obtinrent aucun stuccès chez la Souris. VENGE (I954) ne fut pas plus heureux en utilisant cette méthode ou la précédente. Au contraire, EDWARDS (I954, I958 a et $b$ ) rétussit à obtenir des embryons de Souris tri- et tétraplö̈des en injectant de la colchicine à un moment connu après l'ovulation (ceci en réglant l'heure de l'ovulation par injections d'hormones gonadotropes).

Mais, comme 1'ont constaté plusieurs auteurs, notamment EDWARDS et SIRLIN (I959), les anomalies introduites par la colchicine sont nombreuses : rejet du pronucleus mâle, rejet de tout ou partie de la chromatine femelle, formation de subnuclei. Enfin et surtout par l'injection intrapéritonéale ou intra-utérine de colchicine, la proportion des ovocytes fécondés qui retiennent leur deuxième globule polaire varie d'une expérience à l'autre, de telle sorte que l'analyse éventuelle des descendants est pleine d'incertitude. Plus récemment (I96I), en utilisant la colcémide, EDWARDs n'a pas éliminé ces difficultés. 


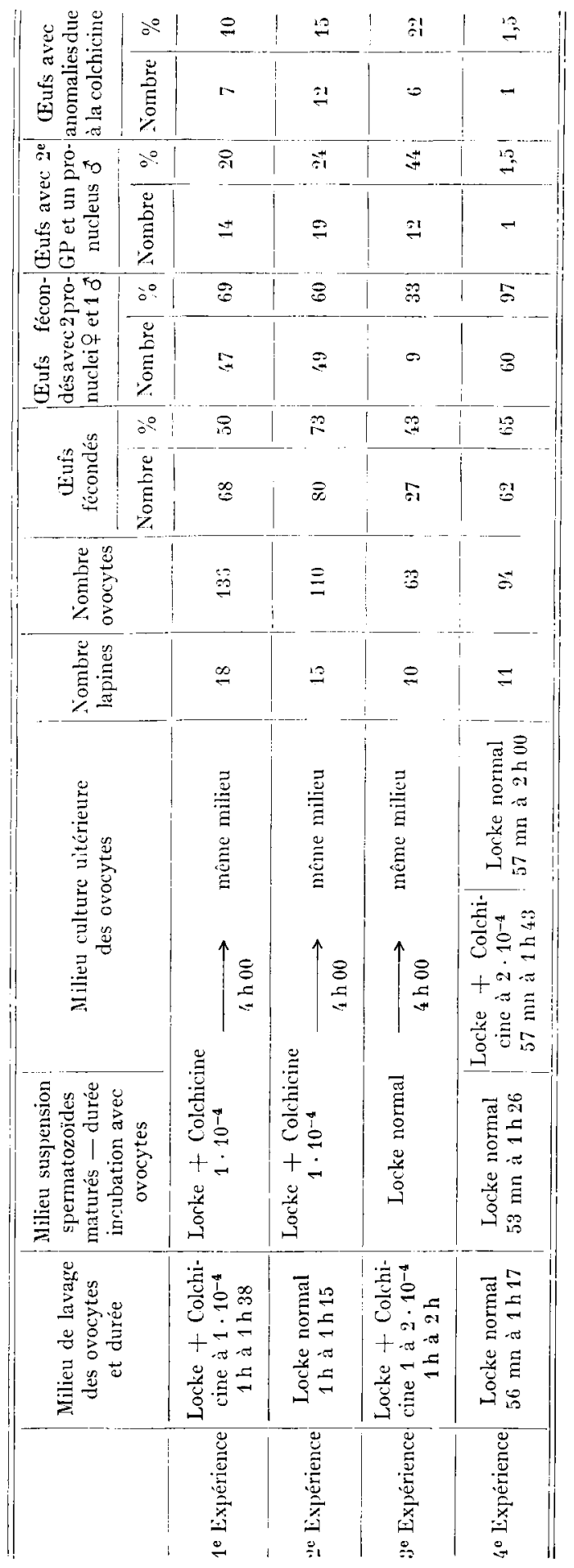


Grâce à la technique de la fécondation in vitro de l'œuf de Lapine (DAUzIER et 'Thibaulí, i959; 'Thibault et Dauzier, I96I), il est possible maintenant de faire agir la colchicine ou la colcémide pendant un temps très précis au cours de la fécondation. Dans les expériences rapportées ici, les ovocytes vierges et les spermatozoïdes maturés sont obtenus selon les méthodes déjà décrites (DAUZIER et THIBAUL,T, I959).

403 ovocytes provenant de 54 Iapines ont été utilisés dans les conditions indiquées dans le tableau.

Rappelons que le lavage préalable des ovocytes fraîchement pondus est une condition essentielle pour la réussite de la fécondation in vitro.

Les deux premières tentatives conduisirent à une proportion assez élevée d'œufs triploïdes : ( 2 noyaux femelles et un noyau mâle), 69 et 60 p. Ioo respectivement. Cependant 20 p. Ioo et 24 p. Ioo des oufs traités, avaient émis leur deuxième globule polaire, Io p. Ioo et I 5 p. Ioo présentaient les anomalies décrites par les auteurs précédents.

L'utilisation d'une telle population d'œufs rendait donc toujours aléatoire l'étude de leurs descendants car, outre les triploïdes, certains œufs sont diploïdes normaux, d'autres diploïdes par élimination du pronucleus mâle, et quelques-uns enfin haplö̈des par élimination après fécondation de la totalité de la chromatine femelle.

La présence d'œufs diploïdes laissait penset que la colchicine n'agissait pas au moment optimum, qui est celui suivant immédiatement la pénétration du spermatozoïde. De plus, la présence d'anomalies pourrait être liée à une action trop prolongée de la colchicine. C'est pourquoi nous avons cherché à faire agir la colchicine seulement au moment même de la fécondation.

On sait qu'in vitro ( Thibaulit et Dauzier, I96I), la fécondation se produit I $h$ zo environ après l'introduction des spermatozoïdes auprès des ovocytes, pourvu que ceux-ci soient lavés auparavant.

En faisant agir la colchicine une heure après l'introduction des spermatozoïdes et pendant une durée d'une heure environ, nous avons obtenu pratiquement Ioo p. IoO d'ovocytes triploïdes sans anomalies (tableau : $4^{\mathrm{e}}$ expérience).

La troisième expérience confirme la nécessité d'assurer une concentration suffisante de colchicine au moment précis de la pénétration du spermatozoïde puisque, si la colchicine est introduite seulement au cours du lavage préalable des ovocytes, il n'y a que 33 p. roo d'œufs triploïdes.

Enfin les expériences I et 3 nous montrent que le séjour des ovocytes dans des solutions de colchicine à $\mathrm{I} \cdot \mathrm{IO}^{-4}$ et spécialement à $2 \cdot \mathrm{ro}^{-4}$ pendant 2 heures avant la fécondation diminue les chances de fécondation, 43 et 50 p. roo (contre 73 et $65 \mathrm{p}$. Ioo) et peut entraîner un pourcentage élevé d'anomalies ( $22 \mathrm{p}$. Ioo, expérience 3 ).

En conclusion, en utilisant la fécondation in vitro qui permet des interventions chronologiquement précises et limitées nous avons mis au point une technique pour obtenir à coup sûr l'obtention d'œufs triplö̈des, par rétention du deuxième globule polaire. 


\author{
SUMMARY \\ IN VITRO FERTILIZATION IN A MEDIUM CONTAINING COLCHICINE \\ AND EXPERIMENTAL POLYPLOIDY IN THE RABBIT
}

For many years, colchicine, and more recently colcemide, are known to induce triploidy in mammals by suppressing the expulsion of the second polar body. But a variable proportion of eggs escape the colchicine action, while abnormalities such as the extrusion of the male pronucleus often occurs.

This paper relates experiments of in vitro fertilization which enabled the authors regularly to obtain triploid eggs without abnormalities, provided colchicine was introduced just before penetration, and was on an average removed one hour later (table : $4^{\text {th }}$ experiment).

This result allows to enter upon a study of the development of triploid embryos resulting from treated eggs transferred into a foster mother.

\title{
RÉFÉRENCES BIBLIOGRAPHIQUES
}

Dauzier L., Thibault C., I959. Données nouvelles sur la fécondation in vitro de l'œuf de la Lapine et de la Brebis.' C. R. Acad. Sci., 248, 2655-2656.

Edwards R. G., I954. Colchicine induced heteroploidy in early mouse embryos. Nature, 174, 276.

EDWARDS R. G., I958 a. Colchicine induced heteroploidy in the mouse. I. The induction of triploidy by treatment of the gametes. J. Exp. Zool., 137, 317-348.

EDWARDS R. G., I 958 b. Colchicine induced heteroploidy in the mouse. II. The induction of tetraploidy and other types of heteroploidy. J. Exp. Zool., 137, 349-362.

Edwards R. G., Sirlin J. L., I959. Identification of C14-labelled male chromatin at fertilization in colchicine-treated mouse eggs. J. Exp. Zool., 140, 19-27.

EDwards R. G., I96r. Induced heteroploidy in mice : effect of deacetylmethylcolchicine on eggs at fertilization. Exp. Cell. Res., 24, 615-617.

HAGgvist G., Bane A., I950 $a$. Chemical induction of polyploid breeds of mammals. Svenska Vet. Akad. Handl., 1, 1-12.

Haggvist G., Bane A., I950 b. Studies in triploid rabbits procuded by colchicine. Hereditas, 36, 329-334.

Melander Y., 1950. Chromosome behaviour of a triploid adult rabbit as produced by H. and B. after colchicine treatment. Hereditas, 36, 335-34I.

Melander Y., I951. Polyploidy after colchicine treatment of pigs. Hereditas, 37, 288-289.

Porpaczy A., Gyorffy B., Farago M., VaskutTi L., 1959. Experiments on treatment of sheep with colchicine (Hongrois). Kyserletuggi Kozlemerujek, 4, 91-100.

Thibault C., Dauzier L., I96r. Analyse des conditions de la fécondation in vitro de l'œuf de la Lapine. Ann. Biol. anim. Bioch. Biophys., 1, 277-294.

VENGE O., I954. Experiments on polyploidy in the rabbit. Kungl. Lantbrukshögskoland Ann., 21, 4I 7 444.

Watdo C. M., Wimsatt W. H., 1945. The effect of colchicine on early cleavage of mouse ova. Anat. Rec., $93,363-373$ 